\title{
Data Inconsistency and Incompleteness Processing Model in Decision Matrix
}

\author{
Daji ERGU ${ }^{1}$, Gang KOU ${ }^{2}$ \\ ${ }^{1}$ School of Management and Economics, \\ University of Electronic Science and Technology of China, \\ Chengdu, 611731, China, \\ ergudaji@163.com \\ ${ }^{2}$ School of Business Administration \\ Southwest University of Finance and Economics of China, \\ Chengdu, 610074, China, \\ kougang@swufe.edu.cn
}

\begin{abstract}
Data Inconsistency and incompleteness issues of pairwise comparison matrix (PCM) are hot research topics in multi-criteria decision making (MCDM). The goal of this paper is to propose a simple approach to identify and adjust the inconsistent data while estimate the missing data in a PCM. Specifically, an arithmetic mean matrix is induced to identify the most inconsistent data efficiently while preserving most of the original information in a PCM, and then we adapt it to estimate the missing data in an incomplete PCM. The proposed model is only dependent on the data in the original matrix, and can effectively process the most inconsistent or missing data in a PCM. The correctness of the proposed method is proved mathematically. Two numerical examples are used to illustrate the proposed method. The result shows that the proposed method is accurate and efficient when processing the inconsistent or missing data to satisfy the consistency requirements of PCM.
\end{abstract}

Keywords: Inconsistency data, Missing data, Pairwise comparison matrix, Arithmetic mean induced bias matrix.

\section{Introduction}

Over the past few decades, the pairwise comparison technique, originally proposed by Thurstone [1], is extensively used to deal with tangible and intangible criteria in multi-criteria decision making (MCDM) methods, especially in the Analytical Hierarchy Process (AHP) and the Analytical Network Process (ANP) [2-9]. All results of $n$ numbers of being compared criteria or alternatives are arranged in a comparison matrix $A=\left(a_{i j}\right)_{n \times n}$, where $a_{i j}>0, a_{i j}$ $=1 / a_{j i}$ and popularly termed pairwise comparison matrix (PCM hereinafter) in literature. The PCM is built to assign criteria weights or scores of alternatives, and is composed of data expressed on a numerical scale (e.g. Saaty's fundamental 9-point scale) and given by decision makers or surveyed experts based on their experiences and expertise. A PCM is said to be perfectly consistent if the expression $a_{i j}=a_{i k} a_{k j}$ holds for all $i, j$ and $k$. However, as the surveyed experts are often biased in their subjective comparisons, a PCM is usually difficult to satisfy the perfectly consistency condition, indicating that the inconsistent comparisons of preference judgment may exist in a PCM. Therefore, the inconsistency issue in a PCM has been widely studied, and a number of approaches and models are proposed and developed [10-15].
Currently, the consistency ratio (CR) proposed by Saaty [16] is widely used to test the consistency of a PCM.

If $C R<0.1$, then the PCM is said to be of acceptable consistency, otherwise, the inconsistent entries should be revised.

In addition to the inconsistent issue, a PCM could also be incomplete due to the large number of criteria being compared (or alternatives), time pressure, lack of the expertise or incomplete information as well as the complexity nature of the decision problem [17]. Therefore, the issue of processing missing data in a PCM has been another hot research topic in the study of multi-criteria decision making (MCDM), and many models are proposed to handle this issue [18-20]

To identify the inconsistent elements simply and accurately while preserving most of the original comparison information in the PCM, an induced bias matrix (IBM) model, which is only based on the original comparison matrix, is proposed in [15]. Ergu and Kou [17] extended the IBM model to process the missing data in a PCM. In this paper, we borrow the concept of the IBM model and propose an arithmetic mean induced bias matrix (AMIBM) model to identify and adjust the most inconsistent elements. Different from the IBM model proposed in [15] and [17], the most 
inconsistent data can easily be identified by observing the largest negative entry in the AMIBM C. Again, we also extend the proposed AMIBM to process the missing data in a PCM, and the missing data are estimated by optimization method.

The rest of this paper is organized as follows. In Section 2, the theorems and corollaries of AMIBM model for inconsistency is proposed and proved mathematically. The theorem of AMIBM is extended to estimate the missing data. The processes of inconsistency identification and the procedures of missing data estimation are further proposed in this section. Two numerical examples are used to illustrate the proposed method for inconsistency identification and missing comparisons estimation in Section 3. A brief conclusion is presented in Section 4.

\section{Arithmetic Mean Induced Bias Matrix (AMIBM) Model}

\subsection{The theorem of AMIBM}

In [15], a bias matrix is induced to amplify the most inconsistent entry of a PCM, and the largest value of the absolute bias data is employed to identify the most inconsistent data. To make the concept of inducing bias matrix more clearly, an arithmetic mean bias matrix is induced to reflect the differences between the arithmetic mean of the indirect judgments $a_{i k} a_{k j}$ and the direct judgment $a_{i j}$. And the most inconsistent data can be identified by observing the largest negative entry of the induced bias matrix C. The proposed theorem is described in more-depth below.

Theorem 1: The arithmetic mean induced bias matrix (AMIBM) should be (or close to) zero matrix if the pairwise comparison matrix (PCM) is perfectly (or approximately) consistent.

$$
C=\frac{1}{n} A A-A=\left(c_{i j}\right) \begin{cases}=0 & \text { if } a_{i k} a_{k j}=a_{i j} \\ \approx 0 & \text { if } a_{i k} a_{k j} \approx a_{i j}\end{cases}
$$

where A represents a PCM, $\mathrm{n}$ denotes the order of matrix A, while $c_{i j}=\frac{1}{n} \sum_{k=1}^{n} a_{i k} a_{k j}-a_{i j}$ is a bias entry located at the ith row and jth column of the arithmetic mean induced bias matrix $\mathrm{C}$.
Proof: According to the consistency condition, the expression $a_{i j}=a_{i k} a_{k j}$ holds for all $i, j, k$ if the PCM is perfectly consistent, then we have,

$c_{i j}=\frac{1}{n} \sum_{k=1}^{n} a_{i k} a_{k j}-a_{i j}=\frac{1}{n} \sum_{k=1}^{n} a_{i j}-a_{i j}=\frac{1}{n} n a_{i j}-a_{i j}=0$

Obviously, the approximately consistent case can easily be proved by replacing the equality symbol "=" with the approximated symbol " $\approx$ ".

Corollary 1: The induced arithmetic mean bias matrix $\mathrm{C}$ can not be a zero matrix if matrix $\mathrm{A}$ is inconsistent. More precisely, there is one entry in $i^{\text {th }}$ row or column of matrix $\mathrm{C}$ greater than 0 .

Proof: If matrix A is inconsistent, Saaty [16] proved that for the maximal eigenvalue $\lambda_{\max }$ of $\mathrm{A}$, the inequality $\lambda_{\max }>n$ holds. In addition, the corresponding unique eigenvector $\omega_{\max }$ is a positive vector.

Applying the following equation to matrix $\mathrm{C}$

$$
A \omega_{\max }=\lambda_{\max } \omega_{\max }
$$

we get

$$
\begin{aligned}
C \omega_{\max } & =\left(\frac{1}{n} A A-A\right) \omega_{\max } \\
& =\frac{1}{n} A A \omega_{\max }-A \omega_{\max } \\
& =\frac{1}{n} A \lambda_{\max } \omega_{\max }-\lambda_{\max } \omega_{\max } \\
& =\frac{1}{n} \lambda_{\max }^{2} \omega_{\max }-\lambda_{\max } \omega_{\max } \\
& =\frac{1}{n} \lambda_{\max }\left(\lambda_{\max }-n\right) \omega_{\max }
\end{aligned}
$$

Since $\lambda_{\max }>n, C \omega_{\max }$ is a positive vector. Consequently, $C$ cannot have any row containing only zeros. More precisely, any row of $C$ must contain at least one positive element.

Corollary 2: The corresponding bias entry $\mathrm{c}_{\mathrm{ij}}$ of matrix $\mathrm{C}$ is less than zero if the inconsistent entry $a_{i j}$ of matrix $A$ is larger than it's theoretically correct value.

Proof: Assume $a_{i j}$ is the most inconsistent entry of matrix A, and is larger than it's theoretically correct value, i.e. 


$$
\left\{\begin{array}{c}
a_{i 1} a_{1 j}<a_{i j} \\
a_{i 2} a_{2 j}<a_{i j} \\
\vdots \\
a_{i n} a_{n j}<a_{i j}
\end{array}\right.
$$

Adding all the inequalities together in the system of inequalities (4), we get

$$
\begin{aligned}
& \sum_{k=1}^{n} a_{i k} a_{k j}<n a_{i j} \\
& \Rightarrow \frac{1}{n} \sum_{k=1}^{n} a_{i k} a_{k j}<a_{i j} \\
& \Rightarrow c_{i j}=\frac{1}{n} \sum_{k=1}^{n} a_{i k} a_{k j}-a_{i j}<0
\end{aligned}
$$

Similarly, if the inconsistent entry $a_{i j}$ of matrix A is smaller than it's theoretically correct value, then its reciprocal entry $a_{j i}$ of matrix A will be larger than it's theoretically correct value, and the corresponding bias entry $c_{j i}$ of matrix $\mathrm{C}$ will also be less than zero.

\subsection{The processes of inconsistency identification and adjustment}

For inconsistency identification, some researchers regarded the largest absolute value(s) in their models as the most inconsistent element $[5,16,21]$. In [15], the most inconsistent entry is also determined by analyzing the absolute largest value in the induced bias matrix C. According to Corollary 2 , if the most inconsistent entry $a_{i j}$ of matrix A is larger (or smaller) than it's theoretically correct value, the value of the corresponding bias entry $c_{i j}$ (or $c_{j i}$ ) of matrix $\mathrm{C}$ will be negative, indicating the average mean of the indirect judgment $a_{i j}=a_{i k} a_{k j}$ is less than the value of direct judgment $a_{i j}$. Therefore, we can define the entry with the largest negative value in the arithmetic mean induced bias matrix (AMIBM) $\mathrm{C}$ as the most inconsistent entry of the original matrix A. The specific steps of inconsistency identification can be summarized as follows:

Step 1: Construct the AMIBM C by eq. (1).

Step 2: Identify the largest bias values with minus signs $c_{i j}^{-\max }$ in matrix $\mathrm{C}$.
Step 3: Determine the corresponding entry $a_{i j}$ of matrix $A$ as the most inconsistent entry.

Step 4: Estimate $a_{i j}$ using the estimation eq. (12).

Step 5: Test the consistency of the revised matrix A.

If the revised matrix A fails to consistency test, then go back to Step 1 above or continue to select the following second, the third largest values with minus sign in matrix $\mathrm{C}$ one by one to revise the matrix $A$ until it meets the consistency condition.

Once the most inconsistent entry is determined, it should be effectively estimated and adjusted. According to [22], the arithmetic mean $\widetilde{a}_{i j}=\frac{1}{n-2} \sum_{k=1}^{n} a_{i k} a_{k j}$ can be used to estimate the inconsistent entry $a_{i j}$, where $i \neq k \neq j$. By eq. (1), the expression $a_{i j}=\frac{1}{n} \sum_{k=1}^{n} a_{i k} a_{k j}$ holds if the original matrix $A$ is perfectly consistent. Here we have assumed $a_{i j}$ as the most inconsistent entry, but the formula $\frac{1}{n} \sum_{k=1}^{n} a_{i k} a_{k j}=c_{i j}+a_{i j} \quad$ contains the inconsistent value of $a_{i j}$ twice when $k=i, j$ (i.e., $a_{i i} a_{i j}=a_{i j}$ and $a_{i j} a_{i j}=a_{i j}$ since $\left.a_{i i}=1, a_{j j}=1\right)$. Therefore, we remove the two $a_{i j}$ from the above formula and use the arithmetic mean $\widetilde{a}_{i j}=\frac{1}{n-2} \sum_{k=1, \neq i, j}^{n} a_{i k} a_{k j}$ to estimate the most inconsistent entry of matrix A. According to the definition of the arithmetic mean induced bias matrix $\mathrm{C}$, the above formula is equivalent to the following formula,

$$
\begin{aligned}
& c_{i j}^{-\max }=\frac{1}{n} \sum_{k=1}^{n} a_{i k} a_{k j}-a_{i j} \\
& \Rightarrow \sum_{k=1}^{n} a_{i k} a_{k j}=n c_{i j}^{-\max }+n a_{i j} \\
& \Rightarrow \sum_{\substack{k=1 \\
k \neq i, j}}^{n} a_{i k} a_{k j}+2 a_{i j}=n c_{i j}^{-\max }+n a_{i j}
\end{aligned}
$$




$$
\begin{aligned}
& \Rightarrow \sum_{\substack{k=1 \\
k \neq i, j}}^{n} a_{i k} a_{k j}=n c_{i j}^{-\max }+(n-2) a_{i j} \\
& \Rightarrow \widetilde{a}_{i j}=\frac{1}{n-2} \sum_{\substack{k=1 \\
k \neq i, j}}^{n} a_{i k} a_{k j}=\frac{n}{n-2} c_{i j}^{-\max }+a_{i j}
\end{aligned}
$$

\subsection{AMIBM for missing data}

In a pairwise comparison matrix $A=\left(a_{i j}\right)_{n \times n}$, there are $n(n-1) / 2$ pairwise comparisons required to be completed. However, when the number of alternatives is large, it is difficult to fill in all $n(n-1) / 2$ pairwise comparisons because of time pressure, lack of related information or preference conflicts, unwillingness to make a direct comparisons between alternatives or being unsure of some of the comparisons, even purposely skip some direct comparisons to make fast decision [17, 22]. To estimate the missing comparisons in an incomplete matrix, the following theorem is proposed.

Theorem 2: The arithmetic mean induced bias matrix (AMIBM) $\varepsilon$ should be equal (or close) to a zero matrix if the pairwise comparsion matrix A is perfectly (or approximately) consistent,i.e.,

$$
\begin{aligned}
\varepsilon & =\frac{1}{n} A(x) A(x)-A(x) \\
& =\left(\varepsilon_{i j}\right) \begin{cases}=0 & \text { if } a_{i k} a_{k j}=a_{i j} \\
\approx 0 & \text { if } a_{i k} a_{k j} \approx a_{i j}\end{cases}
\end{aligned}
$$

where $A(x)$ represents the revised 'complete' matrix $A$ with unknown variables $x_{1}$ and $1 / x_{1} ; x_{2}$ and $1 / x_{2}$, etc, respectively; while $\varepsilon_{i j}=\frac{1}{n} \sum_{k=1}^{n} a_{i k} a_{k j}-a_{i j}$.

Based on Theorem 2, we can define the following optimization problem as the missing data estimation problem. That is, estimate the missing data by minimizing the average sum of least square error of deviation matrix $\varepsilon$.

$$
\text { Min } f\left(a_{i j}, x\right)=\frac{1}{n(n-1)} \sum_{j=1}^{n} \sum_{i=1}^{n}\left(\varepsilon_{i j}^{2}\right)
$$

Therefore, the processes of estimating the missing data in a PCM can be summarized as follows:

Step 1: Complete the missing comparisons with unknown variables $x_{1}$ and $1 / x_{1} ; x_{2}$ and $1 / x_{2}$; etc.

Step 2: Establish the arithmetic mean induced bias matrix (AMIBM) $\varepsilon$ by eq. (13).

Step 3: Construct the optimization problems by eq.(14) and solve the optimization problem.

Step 4: Test the revised comparison matrix A by replacing the missing comparisons with the estimated values.

\section{Illustrative Examples}

\subsection{Inconsistency identification by AMIBM}

Ergu et al. [15] effectively identified the most inconsistent entries $a_{23}$ and $a_{93}$ in the Example 4 by the proposed seven steps, especially the method of matrix order reduction, and the consistency ratio has been improved from 0.2328 to 0.0732 . In this section, the Example 4 used in [15] is introduced to illustrate the proposed AMIBM model and demonstrate the processes of inconsistency identification and adjustment proposed above.

Example 1. The $9 \times 9$ pairwise comparison matrix $A$ used in [15] is inconsistent with $C R=0.2328>0.1$.

$$
A=\left[\begin{array}{ccccccccc}
1 & 2 & 1 / 2 & 2 & 1 / 2 & 2 & 1 / 2 & 2 & 1 / 3 \\
1 / 2 & 1 & 4 & 1 & 1 / 4 & 1 & 1 / 4 & 1 & 1 / 4 \\
2 & 1 / 4 & 1 & 4 & 1 & 4 & 1 & 4 & 1 / 7 \\
1 / 2 & 1 & 1 / 4 & 1 & 1 / 4 & 1 & 1 / 4 & 1 & 1 / 6 \\
2 & 4 & 1 & 4 & 1 & 4 & 1 & 4 & 6 \\
1 / 2 & 1 & 1 / 4 & 1 & 1 / 4 & 1 & 1 / 4 & 1 & 1 / 3 \\
2 & 4 & 1 & 4 & 1 & 4 & 1 & 4 & 7 \\
1 / 2 & 1 & 1 / 4 & 1 & 1 / 4 & 1 & 1 / 4 & 1 & 1 / 2 \\
3 & 4 & 7 & 6 & 1 / 6 & 3 & 1 / 7 & 2 & 1
\end{array}\right]
$$

Apply the proposed AMIBM model to above PCM and detail the AMIBM-based inconsistency identification and adjustment process below.

\section{Step I: Inconsistency identification}

Step 1: Construct the AMIBM C using eq. (1), we have,

s.t. $1 / 9 \leq x \leq 9$ 


$$
C=\left[\begin{array}{ccccccccc}
0 & -0.2824 & 1.0370 & 0 & -0.0494 & -0.1111 & -0.0503 & -0.1481 & 0.7487 \\
0.8611 & 0 & -2.7500 & 1.7222 & 0.3935 & 1.6389 & 0.3929 & 1.6111 & 0.3598 \\
-0.3829 & 2.5357 & 0 & -0.7659 & -0.2126 & -0.8135 & -0.2130 & -0.8294 & 1.8588 \\
0 & -0.1412 & 0.5185 & 0 & -0.0247 & -0.0556 & -0.0251 & -0.0741 & 0.3743 \\
1.7778 & 1.8056 & 6.2222 & 3.5556 & 0 & 1.5555 & -0.0159 & 0.8889 & -3.2434 \\
0.0556 & -0.0671 & 0.6481 & 0.1111 & -0.0216 & 0 & -0.0225 & -0.0370 & 0.2262 \\
2.1111 & 2.2500 & 7.0000 & 4.2222 & 0.0185 & 1.8889 & 0 & 1.1111 & -4.1323 \\
0.1111 & 0.0069 & 0.7778 & 0.2222 & -0.0185 & 0.0556 & -0.0198 & 0 & 0.0780 \\
0.1243 & -0.8902 & -3.1601 & 0.2487 & 1.2474 & 2.9153 & 1.2685 & 3.8042 & 0
\end{array}\right]
$$

Step 2: Identify the largest bias values with minus signs $c_{i j}^{-\max }$ in matrix C. Here,

$c_{79}^{-\max }=-4.1323$.

Step 3: Determine the corresponding entry $a_{79}$ of matrix A as the most inconsistent entry.

\section{Step II: Inconsistency adjustment}

Step 4: Calculate the inconsistent entry $a_{79}$ using eq. (12). (see§2.2)

$\tilde{a}_{79}=\frac{n}{n-2} c_{79}^{-\max }+a_{79}=\frac{9}{7}(-4.1323)+7=1.6871$

Step 5: Test the consistency of the revised matrix A by replacing the $a_{79}$ and $a_{97}$ with 1.6871 and $1 / 1.6871$. We can get that $\lambda_{\max }=10.7326$, and $C R=0.1483$. Although the $C R$ has been improved from 0.2328 to 0.1483 , the revised matrix $A$ still failed to the consistency, thus continue to adjust the second largest value with minus sign, here $c_{59}^{-2 n d \max }=-3.2334$. By eq. (12), we can get $\widetilde{a}_{59}=1.8299$. Similar to Step 5, the corresponding $\lambda_{\max }$ and $C R$ of the second revised matrix $A_{2}$ can be calculated and are 10.3425 and 0.1149 , respectively. It failed to the consistency test. Continue to adjust matrix A by the third largest value with minus sign, here $c_{93}^{-3 r d \max }=-3.1601$. By eq. (12), we have $\widetilde{a}_{93}=2.9371$. The corresponding $\lambda_{\max }$ and $C R$ of the third revised matrix $A_{3}$ are 10.1228 and 0.0961, respectively. Although the calculated $C R$ is already less than 0.1 , the difference is very small. Therefore, we can continue to test the consistency by the fourth largest value with minus sign, here, $c_{23}^{-4 t h \max }=-2.75$, thus we can derive that $\widetilde{a}_{23}=0.4643$. The $\lambda_{\max }$ and $C R$ of the fourth revised matrix $A_{4}$ are 9.3013 and 0.0258 , respectively. Therefore, the $C R$ has been efficiently improved from 0.2328 to
0.0258. Different from [15], we identified another two most inconsistent entries $a_{79}$ and $a_{59}$ except $a_{93}$ and $a_{23}$. The calculated $C R$ $(0.0258)$ is also smaller than the $C R(0.0732)$ obtained in [15]. For simplicity, we can adjust matrix A by calculating $\widetilde{a}_{79}, \widetilde{a}_{59}, \widetilde{a}_{93}$ and $\widetilde{a}_{23}$ simultaneously since their corresponding bias entries in the AMIBM $\mathrm{C}$ are negative and close to each other.

\subsection{Estimating the missing data by AMIBM}

To illustrate the proposed AMIBM model for estimating the missing data in a PCM, let us assume the following 5-by-5 incomplete matrix A with four missing comparisons, where missing comparisons are denoted by ' $x$ '.

$$
A=\left(\begin{array}{ccccc}
1 & \frac{1}{3} & 2 & \frac{1}{5} & \frac{1}{3} \\
3 & 1 & \times & 2 & \times \\
\frac{1}{2} & \times & 1 & \times & \frac{1}{7} \\
5 & \frac{1}{2} & \times & 1 & \times \\
3 & \times & 7 & \times & 1
\end{array}\right)
$$

Apply the proposed AMIBM model to estimate the missing data in matrix A. Details are as follows.

Step 1: Complete the missing comparisons with unknown variables $x_{1}$ and $1 / x_{1} ; x_{2}$ and $1 / x_{2} ; x_{3}$ and $1 / x_{3} ; x_{4}$ and $1 / x_{4}$, we have,

$$
A(x)=\left(\begin{array}{ccccc}
1 & \frac{1}{3} & 2 & \frac{1}{5} & \frac{1}{3} \\
3 & 1 & x_{1} & 2 & x_{2} \\
\frac{1}{2} & \frac{1}{x_{1}} & 1 & x_{3} & \frac{1}{7} \\
5 & \frac{1}{2} & \frac{1}{x_{3}} & 1 & x_{4} \\
3 & \frac{1}{x_{2}} & 7 & \frac{1}{x_{4}} & 1
\end{array}\right)
$$


Step 2: Establish the arithmetic mean induced bias matrix (AMIBM) $\varepsilon$ by eq. (13).

$$
\varepsilon=\frac{1}{n} A(x) A(x)-A(x)
$$

Step 3: Construct the optimization problems by eq. (14),

Min $f\left(a_{i j}, x_{1}, \quad x_{2}, x_{3}, x_{4}\right)=\sum_{j=1}^{5} \sum_{i=1}^{5} \varepsilon_{i j}^{2}$

$\varepsilon_{i j}=\frac{1}{5} \sum_{n=1}^{5} a_{i k} a_{k j}-a_{i j}$

s.t. $1 / 9 \leq x_{1}, \quad x_{2}, x_{3}, x_{4} \leq 9$

Apply the nonlinear constrained optimization function fmincon in Matlab software to solve the above optimization problem, the detailed values of objective function $f(x)$ in each iteration and the estimated optimal values of four variables are shown in Table 1 and plotted in Figure 1.

Figure 1 shows that the value of objective function $f(x)$ almost remains stable after the $7^{\text {th }}$ iteration. It can be seen from Table 1 that the value of objective function changes at the second decimal fraction part. The estimated optimal values of four missing comparisons are $x_{1}=8.8834, x_{2}=0.8834$, $x_{3}=0.1240$ and $x_{4}=1.4770$.
Table 1 The results of optimization by AMIBM

\begin{tabular}{|l|l|l|l|c|l|}
\hline Iter & $\mathrm{f}(\mathrm{x})$ & Iter & $\mathrm{f}(\mathrm{x})$ & variables & $\begin{array}{l}\text { Estimated } \\
\text { value }\end{array}$ \\
\hline 0 & 1.62875 & 7 & 0.28325 & $x_{1}$ & 8.8834 \\
\hline 1 & 1.01686 & 8 & 0.27078 & $x_{2}$ & 0.8834 \\
\hline 2 & 0.86772 & 9 & 0.26543 & $x_{3}$ & 0.1240 \\
\hline 3 & 0.81077 & 10 & 0.26382 & $x_{4}$ & 1.4770 \\
\hline 4 & 0.60542 & 11 & 0.26175 & & \\
\hline 5 & 0.46349 & 12 & 0.26168 & & \\
\hline 6 & 0.42253 & 13 & 0.26166 & & \\
\hline
\end{tabular}

Step 4: Test the revised comparison matrix A by replacing the four missing comparisons in matrix A with these estimated values, we get $\lambda_{\max }=5.1534$ and $C R=0.0342$. Therefore, the revised pairwise comparison matrix A passes the consistency test, and it can be used to make a decision.

\section{Conclusions}

In this paper, an arithmetic mean induced bias matrix (AMIBM) is proposed and proved mathematically, which is easier to be used in practice than the existing IBMM model. The inconsistent entries can be identified by determining the largest values with negative sign in the arithmetic mean induced bias matrix C. Then an adjusting formula is proposed. The proposed AMIBM for inconsistency is further extended to process the missing data in an

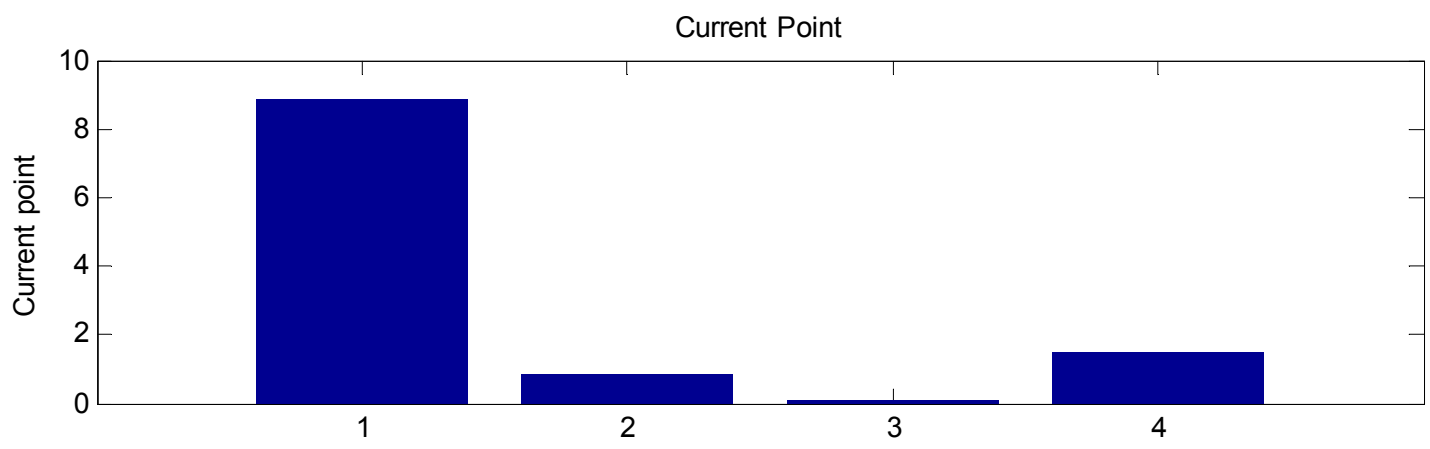

(a) Number of variables: 4

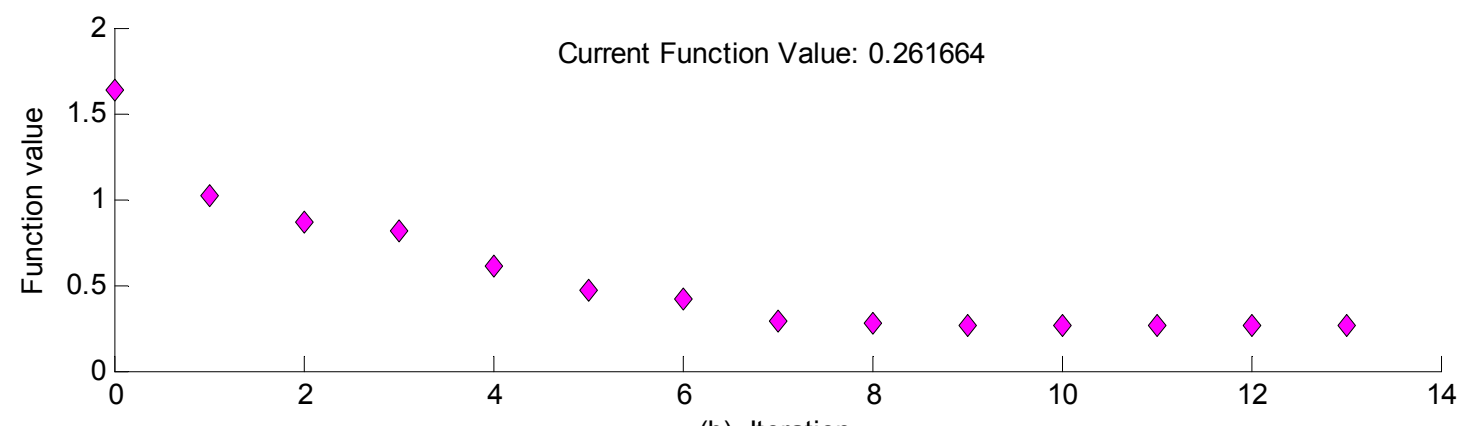

(b) Iteration

Figure 1. Iteration optimization by AMIBM 
incomplete matrix. The proposed AMIBM can deal with the inconsistency issue and the incompleteness issue in a pairwise comparison decision matrix simultaneously. Although the results of two numerical examples show the effectiveness and accuracies of the proposed model, more complicated examples in realworld decision making problem need to be used to test the proposed AMIBM model in future.

\section{Acknowledgements}

This research was partially supported by grants from the National Natural Science Foundation of China \#71222108, \#71173028, \#91224001 and $\# 71373216$.

\section{REFERENCES}

1. THURSTONE, L., Law of Comparative Judgment, Psychological Review, vol. 34, no. 4, 1927, pp. 273-273.

2. SAATY, T. L., Axiomatic Foundation of the Analytic Hierarchy Process, Management Science, vol. 32, No. 7, 1986, pp. 841-855.

3. SAATY, T.L., How to Make a Decision: The Analytic Hierarchy Process, European Journal of Operational Research, vol. 48, no. 1, 1990, pp. 9-26.

4. SAATY, T. L., Some Mathematical Concepts of the Analytic Hierarchy Process, Behaviormetrika, vol. 29, 1991, pp. 1-9.

5. SAATY, T. L., How to Make a Decision: The Analytic Hierarchy Process, Interfaces, vol. 24, no. 6, 1994, pp. 19-43.

6. ERGU, D., G. KOU, Y. PENG, Y. SHI, Y. SHI, The Analytic Hierarchy Process: Task Scheduling and Resource Allocation in Cloud Computing Environment, DOI: 10.1007/s11227-011-0625-1, The Journal of Supercomputing, 2011.

7. PENG, Y., G. KOU, G. WANG, Y. SHI, FAMCDM: A Fusion Approach of MCDM Methods to Rank Multiclass Classification Algorithms, Omega, vol. 39, no. 6, 2011, pp. 677-689.

8. PENG, Y., G. KOU, G. WANG, W. WU, Y. SHI, Ensemble of Software Defect Predictors: An AHP-based Evaluation Method, International Journal of
Information Technology \& Decision Making, vol. 10, no. 1, 2011, pp. 187-206.

9. ERGU, D., G. KOU, Y. PENG, Y. SHI, Y. SHI, Analytic Network Process in Risk Assessment and Decision Analysis, Computers \& Operations Research, vol. 42, 2014, pp. 58-74.

10. XU, Z. S., C. P. WEI, A Consistency Improving Method in the Analytic Hierarchy Process, European Journal of Operational Research, vol. 116, no. 2, 1999, pp. 443-449.

11. ISHIZAKA, A., M. LUSTI, An Expert Module to Improve the Consistency of AHP Matrices, International Transactions in Operational Research, vol. 11, no. 1, 2004, pp. 97-105.

12. LI, H., L. MA, Detecting and Adjusting Ordinal and Cardinal Inconsistencies through a Graphical and Optimal Approach in AHP Models, Computers \& Operations Research, vol. 34, no. 3, 2007, pp. 780-798.

13. CAO, D., L. C. LEUNG, J. S. LAW, Modifying Inconsistent Comparison Matrix in Analytic Hierarchy Process: A Heuristic Approach, Decision Support Systems, vol. 44, no. 4, 2008, pp. 944-953.

14. IIDA, Y., Ordinality Consistency Test About Items And Notation Of A Pairwise Comparison Matrix in AHP, Proceedings of the International Symposium on the Analytic Hierarchy Process, 2009.

15. ERGU, D., G. KOU, Y. PENG, Y. SHI, A Simple Method to Improve the Consistency Ratio of the Pair-wise Comparison Matrix in ANP, European Journal of Operational Research, vol. 213, no. 1,2011 , pp. 246-259.

16. SAATY, T. L., The Analytical Hierarchy Process, New York: McGraw-Hill, 1980.

17. ERGU, D., G. KOU, Questionnaire Design Improvement and Missing Item Scores Estimation for Rapid and Efficient Decision Making, Annals of Operations Research, 2011, DOI: 10.1007/s10479-011-0922-3

18. FEDRIZZI, M., S. GIOVE, Incomplete Pairwise Comparison and Consistency Optimization, European Journal of 
Operational Research, vol. 183, no. 1, 2007, pp. 303-313.

19. GOMEZ-RUIZ, J. A., M. KARANIK, J. I. PELÁEZ, Estimation of Missing Judgments in AHP Pairwise Matrices Using a Neural Network-based Model, Applied Mathematics and Computation, vol. 216, 2010, pp. 2959-2975.

20. BOZÓKI, S., J. FÜLÖP, L. RÓNYAI, On Optimal Completion of Incomplete Pairwise Comparison Matrices,
Mathematical and Computer Modelling, vol. 52, no. 1-2, 2010, pp. 318-333.

21. HARKER, P. T., Alternative Modes of Questioning in the Analytic Hierarchy Process, Mathematical Modelling, vol. 9, no. 3-4, 1987, pp. 335-360.

22. SIRAJ, S., Preference Elicitation from Pairwise Comparisons in Multi-criteria Decision Making, Doctoral dissertation, University of Manchester, 2011. 